\title{
¿Cómo se diseña un curso de creación de materiales?
}

\author{
How it is a material building course designed?
}

\author{
MARÍA SABAS ELÍAS \\ HeRMANDADES DEL TRABAJO CENTRO de MADRID _ CSB ESCUELA DE ESPAÑOL \\ m_sabas@hotmail.com
}

\begin{abstract}
Resumen
La acción formativa de cualquier profesional que se dedica a la enseñanza de lenguas supone un gran valor para la mejora continua de la calidad de su trabajo, así como para el desarrollo de su trayectoria profesional. Y esta formación no solo se circunscribe al docente de lenguas sino también al formador de formadores, al editor y a los autores de materiales didácticos. En este artículo se va a realizar una reflexión acerca de la importancia que tienen en el sector de la enseñanza-aprendizaje de las lenguas los cursos de formación de creación de materiales. Se empezará por exponer la definición del término "material" y sus características. A continuación, se profundizará en la relevancia de estos cursos en el ámbito formativo, así como el tipo de usuario a quien van dirigidos y sus necesidades, la tipología de cursos actual y, por último, los factores a tener en cuenta en el diseño de los cursos de elaboración de material didáctico.

Palabras clave: aprendizaje en línea, creación, adaptación y análisis de materiales, creatividad, formación, material didáctico, Plan Curricular del Instituto Cervantes.
\end{abstract}

\begin{abstract}
The educational action of any professional involved in the teaching of languages, is of great value for the continuous improvement of the quality of his work, as well as for the development of his professional career. This training is not only limited to the language's teacher, but also to the trainer, the editor, and the authors of teaching materials. This article will reflect on the importance of training courses for the creation of materials, in language teaching and learning. It will begin by exposing the definition of the term "material" and its characteristics, then it will delve into the relevance of these courses in the training area, as well as the type of user to whom they are directed and their needs, the current typology of courses and finally, the factors to be considered in the design of the courses for the development of didactic material.
\end{abstract}

Keywords: e-learning, creation, adaptation and analysis materials, training, creativity, teaching materials, Curriculum Plan of the Cervantes Institute. 


\section{Introducción}

En el contexto actual de la didáctica del español como lengua extranjera (ELE, en adelante) es necesario que el profesional de ELE tenga la posibilidad de formarse específicamente en las competencias vinculadas a crear situaciones de aprendizaje, dado que estas situaciones didácticas activan la construcción de aprendizajes significativos y potencian el desarrollo de determinadas competencias en los discentes.

El profesional de ELE tiene la responsabilidad de llevar al aula el material ad hoc para optimizar su competencia docente y el mercado editorial actual le ofrece una gran diversidad de materiales, tanto a través de los manuales como a través de las plataformas digitales.

A continuación, se van a exponer las necesidades que tiene cada tipología de profesional del sector de ELE. El docente interpreta y usa materiales como por ejemplo el manual utilizado por el centro donde desarrolla su labor académica, pero también selecciona, adapta y elabora material didáctico para adecuarlo a la idiosincrasia del aula y, muchas veces, a la retroalimentación de sus estudiantes. El profesor de ELE tiene la responsabilidad de llevar al aula un material didáctico que se adecúe a los objetivos definidos para el curso.

Para ayudar al profesor de ELE en su desarrollo profesional, los cursos de creación de material didáctico tienen como objetivo mejorar la competencia creativa del usuario, es decir, del profesor de ELE, aplicada al diseño, a la adaptación y a la elaboración de materiales.

\section{Definición de material didáctico: concepto y clasificación}

A lo largo de la historia, el material empleado en las clases ha tenido múltiples denominaciones como, por ejemplo, "material de apoyo", "herramienta de aprendizaje", "medios educativos" etc.; sin embargo, sin embargo, el término que se ha impuesto y es actualmente el más utilizado es el de "material didáctico".

Se presentan a continuación las principales definiciones que se han dado de "material didáctico" porque proporcionan el significado más completo del término.

La primera de ellas data de 1972, y su autor -McKean (1972)- define en su libro Principios y Métodos en la Educación Secundaria al material didáctico en los siguientes términos:

[...] como el nexo entre las palabras y la realidad. Lo ideal sería que todo aprendizaje se lleve a cabo dentro de una situación real de vida. No siendo esto posible, el material didáctico debe sustituir a la realidad, representándola de la mejor forma posible, de modo que facilite su objetivación por parte del alumno (McKean, 1972: 282).

En segundo lugar, es interesante destacar la definición propuesta por Skolverket- La organización de la Agencia Nacional Sueca de Educación- que, 
en su informe sobre la función de los materiales didácticos en la enseñanza, (Skolverket, 2006b) utiliza el concepto de material didáctico en un sentido muy amplio, y usa la definición que hay en el Plan de estudios para la escuela obligatoria en Suecia del año 1980, (Lgr 80: Läroplan för grundskolan). Según esta definición, un material didáctico no es necesariamente algo producido para la enseñanza, es la intención del profesor la que decide si un determinado documento puede convertirse en material didáctico, por lo que cualquier recurso puede utilizarse con esta finalidad.

Por su parte, Boel Englud (2006: 4-6), propone una clasificación del material didáctico en cinco categorías: la primera comprendería los libros publicados por editoriales especializadas en la didáctica de ELE; la segunda, los diccionarios y libros especializados; la tercera, todo aquello que puedan proporcionar los medios de comunicación -incluyendo las TIC-; la cuarta, la "realidad" -por ejemplo, las experiencias propias de docentes y discentes- $y$, por último, los medios audiovisuales.

Por último, Pablo Alberto Morales (2012), en su obra Elaboración de material didáctico, lo define con estas palabras:

el conjunto de medios materiales que intervienen y facilitan el proceso de enseñanza-aprendizaje. Estos materiales pueden ser tanto físicos como virtuales (Morales, 2012: 10).

En este artículo se va a utilizar como referencia la definición amplia del concepto de materiales didácticos que se usa en Lgr 80. La interpretación del concepto de material didáctico de Skolverket (2006) provoca una interesante reflexión acerca de cuáles serían las particularidades que convierten en didáctico a un determinado material.

\section{Características del material didáctico y objetivos de los cursos de creación de materiales.}

Con el fin de determinar qué características debe tener un material para llegar a ser didáctico se toman como referencia los criterios postulados por Tomlinson (2010). Según este autor un material será óptimo en la medida en que coopere a impulsar los procesos de enseñanza-aprendizaje

Las características mínimas que Tomlinson (2010, citado por Ezeiza, J., 2007: 50-51) considera que deben poseer son las siguientes:

- debe producir un efecto positivo en el alumno, motivarlo, generar confianza en el proceso de aprendizaje,

- debe ser provechoso, convertir al estudiante en un agente activo,

- debe facilitar el uso natural y auténtico de la lengua,

- debe potenciar el objetivo comunicativo del aprendizaje de la lengua,

- debe valorar el tiempo necesario para que el aprendiente sea competente, 
- debe tener en cuenta la variedad de estilos de aprendizaje,

- debe poner en valor el componente afectivo,

- debe respetar los periodos de silencio necesarios al comienzo del contacto con la lengua meta,

- debe optimizar la capacidad de análisis y de creatividad del discente y, como colofón,

- debe estimular la retroalimentación como estrategia de aprendizaje.

Los cursos de creación de materiales deben estar orientados a conseguir que los profesores de ELE sepan cómo crear o modificar un material determinado para que, tomando en consideración el tipo de alumnos a los que va dirigido, reúna las características que se han expuesto en el apartado anterior. La existencia de cursos de creación de materiales de didáctica de ELE es esencial para poder facilitar a los profesionales las herramientas idóneas para poder realizar la selección, la adaptación y la creación de materiales didácticos con rigor metodológico, actitud crítica y reflexiva y, de este modo, favorecer el desarrollo profesional de estos.

Los cursos de creación de material didáctico facilitan la posibilidad de realizar un análisis cualitativo del material que nos ofrece el mercado editorial. Siguiendo las investigaciones de Ezeiza (2009) sobre material didáctico, a continuación, se enumeran los principales valores que el autor destacó en estos que constituyen, por tanto, una guía de criterios aplicable al análisis del material didáctico, muy especialmente al análisis de los manuales:

- Los manuales constituyen una gran ayuda en aquellas situaciones en las que los alumnos no cuentan con la posibilidad de mantener contacto directo con la segunda lengua.

- Muchas actividades y materiales recogidos en ellos responden a necesidades generales que afectan, prácticamente, a todos los estudiantes.

- Los manuales tienen una presentación que responde a los estándares de calidad vigentes en la sociedad $y$, en general, presentan una edición mucho más funcional que cualquier material que pueda confeccionar un profesor para sus alumnos.

- Si se conciben como un "paquete de recursos" -y si son susceptibles de ser adaptados y modificados por los profesores-, pueden resultar una ayuda muy útil; entre otras razones, porque permiten acceder en cualquier momento a un amplio abanico de actividades, contenidos e informaciones.

- Ofrecen una buena orientación a los alumnos. Contribuyen a que éstos visualicen y entiendan la lógica del programa o del itinerario que se les propone; sirven, también, para establecer más claramente qué va a ser evaluado. 
- Pueden ser útiles como referencia para medir los progresos realizados ya que ofrecen a los alumnos una base sobre la que determinar en qué aspectos se está produciendo un avance y qué aspectos pueden requerir algún tipo de refuerzo.

- Ofrecen una base sobre la que articular una posible negociación de los objetivos, los contenidos, los procedimientos o del ritmo de trabajo.

- Pueden facilitar la gestión del aula y la de los procesos de aprendizaje.

- Contribuyen a construir una rutina estructurada que permite ajustar la impredecibilidad de la dinámica interaccional del aula a un nivel tolerable. Esto aporta la seguridad psicológica que alumnos y profesores requieren para participar de forma activa en dicha dinámica interaccional y para ensayar fórmulas que permitan un aprendizaje más rápido y eficaz.

- Ofrecen a otros agentes implicados (instituciones de enseñanza; empresas que subvenciona los cursos de sus trabajadores; instituciones administrativas; padres y madres...) un canal que legitima su intervención en el proceso de enseñanza-aprendizaje.

- Facilitan el aprendizaje autónomo de los alumnos fuera del aula.

- Aligeran la carga de trabajo de los profesores, lo cual les permite dedicar más recursos a la atención personalizada del alumno (Ezeiza, 2009: 7).

El reto de cualquier material didáctico de ELE sería que este lograra aproximarse a las necesidades de los estudiantes en diversos contextos y la solución para conseguirlo consistiría en constituir un marco dinámico en el que (como indica Rubdy, 2003: 41) sea factible un equilibrio entre numerosos factores: condicionantes como lo convencional versus lo idiosincrático de las necesidades sociales, la responsabilidad versus la creatividad, la naturaleza impredecible del aprendizaje versus la consistencia de un programa, las necesidades colectivas versus las individuales y la innovación en el diseño y gestión de los materiales versus el conservadurismo.

Todos los especialistas en el campo de la didáctica de las lenguas comparten el postulado de que los materiales son un excelente instrumento para la formación y el desarrollo profesional del docente. Sin embargo, para poder llegar a canalizar adecuadamente dicho potencial es preciso contar con las herramientas adecuadas para establecer indicadores de calidad que permitan valorar adecuadamente los métodos y los materiales para la enseñanza de ELE. (Comisión Europea, 1996: 68). 


\section{Destinatarios de los cursos de creación de material didáctico en el contexto del español como lengua extranjera}

El proceso de creación de materiales de ELE es muy complejo (puede consultarte, a este respecto, el artículo de Paula Cerdeira, en este mismo volumen de DOBLELE) y abarca a diversas tipologías de profesionales vinculados a la enseñanza del ELE y a la certificación lingüística: docentes, editores y autores de materiales, y formadores en didáctica de la enseñanza del español como lengua extranjera. Todos ellos tienen la necesidad de reflexionar sobre las diversas facetas de su profesión. Un ejemplo de ello sería la reflexión sobre qué tipo de materiales didácticos son apropiados para un entorno lingüístico preciso.

Si bien es cierto que existen unos principios básicos que rigen los criterios que deben tomarse en consideración en el proceso de elaboración de materiales, también lo es el hecho de que las necesidades difieren mucho dependiendo de la tipología del aprendiz. Por consiguiente, es preciso realizar un análisis de las necesidades de cada grupo en cuestión.

\section{Análisis de necesidades de los cursos de creación de materiales}

Actualmente, los profesionales de ELE poseen un amplio abanico de recursos didácticos para atender las necesidades específicas de cada aprendiente gracias a que para cubrir dichas necesidades las empresas del sector editorial ponen al alcance de los usuarios una gran variedad de manuales de español lengua extranjera o segunda lengua.

Cabe destacar, por ejemplo, los manuales de español general (con o sin inmersión lingüística) o aquellos que son destinados a fines específicos (el español de los negocios o el del turismo, etc.). Atendiendo a un criterio geográfico-cultural, destacarían los manuales del español destinados a estudiantes inmersos en un contexto latinoamericano; no es baladí que en cuanto a número de hablantes el español de América sobrepasa y con creces al peninsular.

Teniendo en consideración los criterios clasificatorios de índole lingüística, las editoriales disponen de gramáticas del español, diccionarios monolingües, lecturas graduadas, etc. Teniendo en cuenta la edad del alumno, hay diversidad de material bien para niños, bien para adolescentes y adultos. Por último, y no menos relevante, el mundo editorial ofrece como material didáctico complementario acceso a sus propias plataformas de aprendizaje en formato digital. No obstante, desde 2020 y debido a la crisis sanitaria mundial provocada por el Covid-19, la didáctica ha sufrido un cambio abrupto y rápido: la digitalización de la enseñanza y esto ha traído consigo un cambio de orientación metodológica para conseguir una óptima adaptación de la enseñanza presencial a la virtual.

El material en formato digital ha venido experimentando a raíz de la pandemia un crecimiento vertiginoso. Al comienzo de la crisis sanitaria provocada por el Covid-19 se desencadenó un rápido proceso por el cual el colectivo de docentes comenzó a adaptar el material que usaba hasta 
entonces al nuevo escenario virtual. Posteriormente, a medida que la crisis sanitaria se extendía en el tiempo, las empresas del sector editorial procedieron a elaborar material didáctico innovador y adaptado al formato en línea. Esta situación agudizó, más aún si cabe, la necesidad imperiosa de ampliar la formación de cualquier profesional de ELE que se planteara la creación de material didáctico para esta nueva realidad virtual.

Además de las editoriales los docentes también publican sus propios materiales tanto de elaboración propia como adaptaciones de otros materiales ya publicados. Ellos utilizan diversos canales de publicación, bien a través de las redes sociales -fundamentalmente en formato de webinario, en blogs, tutoriales y plataformas digitales- bien en congresos, jornadas, talleres o cualquier evento vinculado al mundo ELE. Sin embargo, a veces dicho material no cumple los estándares de calidad y rigor necesarios para su implementación. Debido a ello es imprescindible que el profesional de la enseñanza de español como lengua extranjera se recicle formativamente y así pueda mejorar sus competencias y además conocer las innovaciones metodológicas en este campo.

Por tanto, los creadores de cursos de elaboración de materiales partes de estas necesidades formativas. A continuación, se va a reflexionar acerca de las ventajas que ofrecen los cursos de creación de material didáctico a todos los usuarios.

En primer lugar, los cursos les van a proporcionar un acercamiento al conocimiento de los distintos tipos de materiales que existen en el mercado actual. En segundo lugar, les van a ayudar a familiarizarse con los criterios y herramientas de análisis de los materiales. En tercer lugar, les van a permitir conocer el proceso de su diseño y determinar la adecuación de estos para conseguir el desarrollo de las capacidades de comprensión y producción. En cuarto lugar, les van a introducir en los rudimentos de la programación de una unidad didáctica y de sus respectivas actividades.

El profesor de ELE necesita llevar al aula un material didáctico que se adecúe a los objetivos definidos en el currículo del programa. En este contexto se dan diversos escenarios que, a continuación, se detallan. Uno de los escenarios habituales con el que se encuentra el docente es que el centro donde ejerce su labor didáctica tiene un determinado manual establecido para todo el equipo. Es en este marco donde su actividad formativa se extiende y se acerca a la creativa, puesto que tiene la necesidad de adaptar y complementar ese material didáctico a la realidad del aula, teniendo en cuenta factores como el perfil del alumno, su nacionalidad, su edad, su inmersión lingüística, etc.

En otros casos, la elección del material didáctico recae en el propio docente, que suele decantarse por la elaboración de material propio, claro está, siguiendo el Plan Curricular del Instituto Cervantes (en adelante, PCIC; Instituto Cervantes, 2012). Esta selección y elaboración es muy compleja, puesto que para llevarla a cabo el profesor debe sopesar las particularidades propias de la enseñanza/aprendizaje tales como las necesidades de los discentes, el contexto educativo, los objetivos de la docencia y la 
metodología, entre otros, y siempre con la finalidad de facilitar el aprendizaje de la lengua, en este caso, el español.

\section{Habilidades que necesita poseer un creador de materiales didácticos}

Para empezar, el creador de materiales necesita tener una gran capacidad reflexiva y analítica para poder hacer una óptima selección del material, bien auténtico bien adaptado, que incluirá en su material didáctico. Además, capacidades tales como la creatividad y la imaginación están muy relacionadas con el pensamiento crítico. La dimensión creativa constituye una base de apoyo en la enseñanza y el aprendizaje de las lenguas. Por ello, esta capacidad creativa es esencial para el futuro autor de materiales. La creatividad es la capacidad de crear alejándose de los pensamientos y conductas habituales., es el arte de combinar de formas diferentes los conocimientos, es el pensamiento innovador. Albert Einstein (1931, citado por Assmann p. 31) decía que el arte supremo del profesor consiste en despertar la alegría por la expresión creativa y el conocimiento. Por tanto, enseñar es un proceso bidireccional en el cual tanto el docente como el alumno se benefician. El verdadero reto del profesor no consiste en trasmitir sus conocimientos para que los estudiantes adquieran nuevas destrezas, sino en fomentar en los alumnos el pensamiento crítico y la creatividad.

El análisis de diferentes materiales puede ayudar a mejorar la capacidad de pensamiento crítico. Resulta especialmente importante observar los posibles objetivos o los motivos que puede tener una persona para crear dicho material.

Crear y diseñar nuestros propios materiales es una actividad muy motivadora, además de ser una tarea imprescindible en esta profesión porque siempre es necesaria la creación de actividades que complementen y adapten los materiales publicados. Trabajar con materiales ya creados es más complejo de lo que parece a primera vista: para hacer un uso eficiente de ellos se necesita capacidad de reflexión y análisis crítico para seleccionar, modificar, actualizar, corregir, añadir, suprimir, temporalizar y secuenciar.

\section{Tipos de cursos de creación de materiales presentes actualmente en el mercado}

Actualmente existe una gran variedad de cursos de creación de materiales en el mercado. Instituciones como el Instituto Cervantes, las universidades españolas -a través de másteres en didáctica de ELE-, o los centros de enseñanza de ELE ofertan cursos de formación de profesores donde una parte del programa se dedica a la elaboración de materiales. Estos cursos se ofrecen tanto en la modalidad presencial como en la virtual, síncrona y asíncrona. En cuanto a la extensión de los cursos, los hay más breves como, por ejemplo, talleres monográficos o webinarios y de gran extensión y profundización tales como los postgrados o másteres dentro de un curso 
académico. Además de los cursos generales para la elaboración de material didáctico existen otros cursos donde se imparte formación específica acerca de algún determinado aspecto de la didáctica de ELE: la innovación y la creatividad en el aula de segundas lenguas o las TIC (tecnologías de la información y de la comunicación) en la era digital.

Las actividades que se plantean a los alumnos constituyen la base del éxito de las clases. Por eso, en estos cursos se aprende a cómo seleccionar las actividades más adecuadas y motivadoras. Además, se aprende cómo se pueden utilizar los materiales a nuestro alcance, cómo explotarlos y qué finalidad se debe perseguir con ello ya que cualquier material (texto, canción, fotografías, viñetas, películas, conversaciones...) puede ser usado como recurso pedagógico. Para ello hay que clasificarlo en un determinado nivel, adaptarlo (si fuera necesario), trabajarlo con una finalidad concreta y, en algunos casos, crear las actividades significativas para completarlo.

\section{Factores que se deben tener en cuenta en el diseño de un curso de elaboración de materiales}

Para comenzar a abordar esta cuestión tomamos como referencia la reflexión de la profesora Aristu (2020). Según Aristu, los tres aspectos esenciales que constituyen la competencia docente en la creación y diseño de materiales son: los conocimientos teóricos sobre el aprendizaje y la enseñanza de las lenguas, la experiencia en el análisis de materiales y la reflexión.

No se puede obviar el hecho de que la finalidad de los cursos de creación de materiales es lograr desarrollar la capacidad efectiva del profesional de ELE en el ámbito más creativo de su profesión: la elaboración y adaptación de materiales didácticos de español, que es una de las competencias básicas de estos profesionales.

Antes de comenzar con el diseño de un curso de creación de materiales se debería reflexionar sobre las siguientes cuestiones: qué se pretende hacer, cómo se quiere hacer, para quién, para qué y por qué.

El punto de partida sería determinar los objetivos del curso. Al tratarse de un curso de creación de material didáctico los objetivos específicos podrán ser los siguientes: en primer lugar, que el estudiante del curso sea capaz de realizar un análisis profundo y comparativo de los materiales y recursos didácticos para la enseñanza del español como lengua extranjera, en segundo lugar, que sea competente en la adaptación de materiales publicados y finalmente, en tercer lugar, que pueda elaborar y diseñar unidades didácticas.

Una vez fijados los objetivos que se deberían lograr en el curso se seleccionaría los contenidos que se van a abordar para alcanzar sus objetivos. Las destrezas de comprensión y de expresión, las estrategias de aprendizaje, el tipo método o enfoque (funcional, estructuralista, comunicativo, etc.), la interculturalidad, la gamificación, la pragmática, la tipología textual, el contenido cultural, etc. serían los contenidos que se debería tratar en el currículo. Aparte de los anteriores contenidos 
relacionados con el diseño de actividades habría que mencionar otros como la secuenciación, las muestras de lengua, la integración de las destrezas, las áreas temáticas, las dinámicas de grupo, el grado de autonomía del estudiante o el componente emocional.

Después se debería determinar qué metodología se va a utilizar para la impartición del curso y cuáles serían los criterios de evaluación del mismo. A continuación, se definiría la carga lectiva, el perfil del futuro estudiante y demás procedimientos encaminados a conformar el programa formativo. Finalmente, una vez elaborada la estructura del curso -a partir de los objetivos, contenidos, destrezas, etc.-, se procederá a materializar el curso, previa realización de la guía docente.

\section{Conclusión}

Como epílogo a este artículo cabría ahondar en algunas de las principales ideas que se han expuesto. A través de su lectura se ha podido constatar el valor que tienen, en el marco de la didáctica del español lengua extranjera, los cursos de creación de materiales. El alcance de estos cursos no se limita únicamente al docente de ELE ni al formador de formadores, sino que trasciende a otros perfiles profesionales del sector, cuya actividad no es meramente formativa sino de otra índole, tales como el editor, el asesor lingüístico o el adaptador. A pesar de la disparidad de los "usuarios" de los cursos de creación de materiales el contenido suele ser bastante homogéneo con el propósito de poder aunar las necesidades de todos ellos. Se ha puesto también de manifiesto la diversidad de las necesidades de los estudiantes en los distintos entornos de aprendizaje debido principalmente a condicionantes externos (véase, la situación de inmersión, académica, lingüística o de cualquier otra condición), y ello produce innumerables variables a las que cualquier futuro creador debería prestar atención.

En las primeras líneas de este artículo se ha puesto en valor la importancia de la formación continua en el ámbito académico. Y dado que la didáctica de la enseñanza de lenguas, en este caso del español como lengua extranjera, está siempre en constante evolución e innovación todo profesional del sector debería poder ampliar y perfeccionar dicha formación. Si además este profesional deseara profundizar en la parte creativa con el propósito de combinar sus conocimientos de un modo singular, original y diferenciador los cursos de elaboración de materiales serían óptimos para ello.

\section{Bibliografía}

ARISTU, A. (2020): ¿ ¿l profesor de español como creador y diseñador de materiales? UNIBA: Universitat de Barcelona. En línea: https://www.unibarcelona.com/es/actualidad/noticias/el-profesor-deespanol-como-creador-y-disenador-de-materiales

AssmanN, H. (2002): Placer y ternura en la educación. Hacia una sociedad aprendiente. Madrid: Narcea, p. 31. 
Consejo de EuRopa (2001): Marco común europeo de referencia para las lenguas. Madrid: Instituto Cervantes.

ENGLUND, B. (2006): Vad har vi lärt oss om läromedel? Lärarhögskolan i Stockholm.

EZEIZA, J. (2007): “Hacia un modelo de calidad en la producción, selección y aplicación de materiales para la enseñanza de lenguas", Actas del Segundo Congreso Virtual E/LE: "La red como espacio de colaboración". En línea: https://issuu.com/civele/docs/actas2007

EzEIZA, J. (2009): "Analizar y comprender los materiales de enseñanza en perspectiva profesional: algunas claves para la formación del profesorado" MarcoELE, no 9. En línea:

https://marcoele.com/suplementos/analizar-y-comprendermateriales/

FERNÁNDEZ LóPEZ, M. a del C. (2000): "Selección de manuales y materiales didácticos", Cuadernos Cervantes. Madrid. En línea: https://www.cuadernoscervantes.com/ele 27 materiales.html

FERNÁNDEZ LóPEZ, M.a del C. (2014): "Principios y criterios para el análisis de materiales didácticos", en J. Sánchez Lobato e I. Santos Gargallo (eds.), Vademécum para la formación de profesores. Madrid, SGEL, p. 715.

GARMENDIA, A. y SANS, N. (2015): “Diseño de materiales para la enseñanza y el aprendizaje de ELE: 10 preguntas imprescindibles", en Herrera, F. (ed.). La formación del profesorado de español. Cuadernos de didáctica. Barcelona: Difusión.

InStituto CeRVANTES (2008): Diccionario de términos clave de ELE. Madrid, SGEL- Educación. En línea: https://[cvc.cervantes.es/Ensenanza/biblioteca ele/diccio ele/defa ult.htm

Instituto Cervantes (2012): Plan Curricular del Instituto Cervantes. Madrid, Biblioteca Nueva. En línea: https://cvc.cervantes.es/ensenanza/biblioteca ele/plan curricular/

MARTín PERIS, E. (1996): Las actividades de aprendizaje en los manuales de ELE. Tesis doctoral. Barcelona: Universitat Pompeu Fabra. En línea:

https://www.tdx.cat/bitstream/handle/10803/286731/01.\%20EMP 1 de3. pdf? sequence $=1$

Martín Peris, E. (2004): “¿Qué significa trabajar en clase con tareas comunicativas?", redELE. En línea http://www.mec.es/redele/revista/martin.shtml

McKeAN, R. (1972): Principios y Métodos en la Educación Secundaria. Buenos Aires: Troquel.

Morales, P. A. (2012): Elaboración de material didáctico. México: Red Tercer Milenio.

Moreno García, C. (2011): Materiales, estrategias y recursos para la enseñanza del español como 2/L. Madrid, Arco Libros.

RuBdY, R. (2003): "Selection of materials", en B. Tomlinson, (ed.), Developing materials for language teaching. 1st edition. London: Continuum, $\mathrm{p}$. 37. 
SANS, Neus (2000): “Criterios para la evaluación y el diseño de materiales didácticos para la enseñanza de ELE", en Actas del VIII Seminario de Dificultades Específicas de la Enseñanza del Español a Lusohablantes. Brasilia: Consejería de Educación en Brasil (MEC) y Colegio Miguel de Cervantes, pp. 10-22.

SKOLVERKET (2006): Lusten och möjligheten - om lärares betydelse, arbetssituation ochförutsättningar. Fördjupande utvärdering utifrån den nationella utvärderingen 2003 av grundskolans årskurs 9. Skolverkets rapport $n r$ 282. Stockholm, Fritzes kundservice. 